Check for updates

Cite this: RSC Adv., 2017, 7, 27668

Received 27th February 2017

Accepted 16th May 2017

DOI: $10.1039 / c 7 r a 02430 d$

rsc.li/rsc-advances

\section{Reductive dechlorination of endosulfan isomers and its metabolites by zero-valent metals: reaction mechanism and degradation products $\uparrow$}

\begin{abstract}
Swatantra P. Singh 1 i $\ddagger$ and Purnendu Bose*
The widely used organochlorine pesticide endosulfan (ES) is extremely toxic to fishes, other aquatic species and mammals. Current evidence suggests that all known natural attenuation residues of ES, i.e., ESmetabolites, retain the original chlorinated skeleton of ES and are potential carcinogens. The objective of the present study was to identify the dechlorinated products formed during the reduction of ES-isomers, i.e., endosulfan-1 and endosulfan-2 and ES-metabolites, namely endosulfan sulfate (ES-S), endosulfan lactone (ES-L), endosulfan ether (ES-E) and endosulfan alcohol (ES-A), by various metallic surfaces. During dechlorination by nano zero-valent iron (NZVI), the mass spectra of the degradation products were consistent with the loss of one, two and three chlorine atoms from the parent molecules. During the interaction of ES-E with $\mathrm{Mg}^{\circ}$, the degradation products had mass spectra consistent with the loss of 1-6 chlorine atoms from the parent molecule. In all cases, dechlorination appears to occur through sequential electron transfer at the metallic surface. Tentative chemical structures for various degradation products were proposed. Synthesis of some of the observed degradation products was possible using ES-E as the starting molecule. ${ }^{1} \mathrm{H}$ NMR, crystallographic (X-ray diffraction) and GC-MS analysis of the synthesized products provide final confirmation of the proposed chemical structures. A pathway for stepwise reductive dechlorination of ES-isomers and metabolites is proposed. Some of the degradation products identified in the present study being less chlorinated, may be less toxic and more amenable to subsequent biodegradation and ultimate mineralization in the natural environment.
\end{abstract}

\section{Introduction}

The organochlorine pesticide endosulfan (ES) has been widely used since the 1950s. ${ }^{1,2}$ Commercial grade ES is available as a $7: 3$ mixture of the two isomers, endosulfan-1 (ES-1) and endosulfan-2 (ES-2). ES is an endocrine disrupter with a primary effect on the central nervous system (CNS). It is very toxic to fishes and other aquatic species ${ }^{3-5}$ and also reported to cause mammalian gonadal toxicity, genotoxicity, and neurotoxicity. ${ }^{6,7}$ ES-isomers were included in the list of POPs (Persistent Organic Pollutants) in the Stockholm convention of $2011 .^{8}$ Natural attenuation of ES through hydrolysis, photo-degradation and biodegradation leads to the formation of degradation products, i.e., ES-metabolites (Fig. 1), such as, endosulfan sulfate (ES-S), endosulfan lactone (ES-L), endosulfan ether (ES-E) and

Department of Civil Engineering, Indian Institute of Technology, Kanpur-208016, India. E-mail: pbose@iitk.ac.in; Tel: +91-512-2597403

$\dagger$ Electronic supplementary information (ESI) available: Supplementary data has 17 pages (S1-S17), 9 Figures (SI1-SI9), and 7 Tables (SI1-SI7). CCDC 1534755. For ESI and crystallographic data in CIF or other electronic format see DOI: 10.1039/c7ra02430d

\$ Present address: Department of Desalination and Water Treatment, Zuckerberg Institute of Water Research, Ben-Gurion University of Negev, Sede-Boqer-89990, Israel. endosulfan alcohol (ES-A). ${ }^{9-14}$ All known natural attenuation residues of ES retain the original chlorinated ring structure of ES and are toxic and potential carcinogens. ${ }^{15}$ There is no concrete evidence in literature that natural attenuation through chemical or aerobic/anaerobic biological processes can disrupt the chlorinated ring structure of ES, i.e., the ES skeleton. Studies using $\mathrm{C}^{14}$-labelled ES have shown that the rate of mineralization of ES by natural attenuation processes is negligibly small. ${ }^{\mathbf{9}, 16}$

Henschler ${ }^{17}$ did a comprehensive review of the toxicity of poly-chlorinated compounds and concluded that the toxicity of poly-chlorinated compounds was directly related to the number of chlorine atoms in the compounds. ${ }^{18}$ Hydrophobicity and stability of chlorinated compounds generally increases with chlorine atoms ${ }^{\mathbf{1 9 2 0}}$ and this leads to higher accumulation and persistency of poly-chlorinated compounds in the environment. A computational study by Zahedi-Tabrizi and Farahati (2011) reported an increase in the strength of intermolecular hydrogen bonding with increase in the number of chlorine atoms in a compound..$^{21}$ Biodegradation of poly-chlorinated compounds is difficult because of their stability and toxic nature. ${ }^{22,23}$ Furukawa et al. related the degree of chlorination in 31 isomers of poly-chlorinated biphenyl (PCBs) to their biodegradation potential and found an inverse relation between biodegradation potential and the number of chlorine atoms in the molecule. ${ }^{22,23}$ 


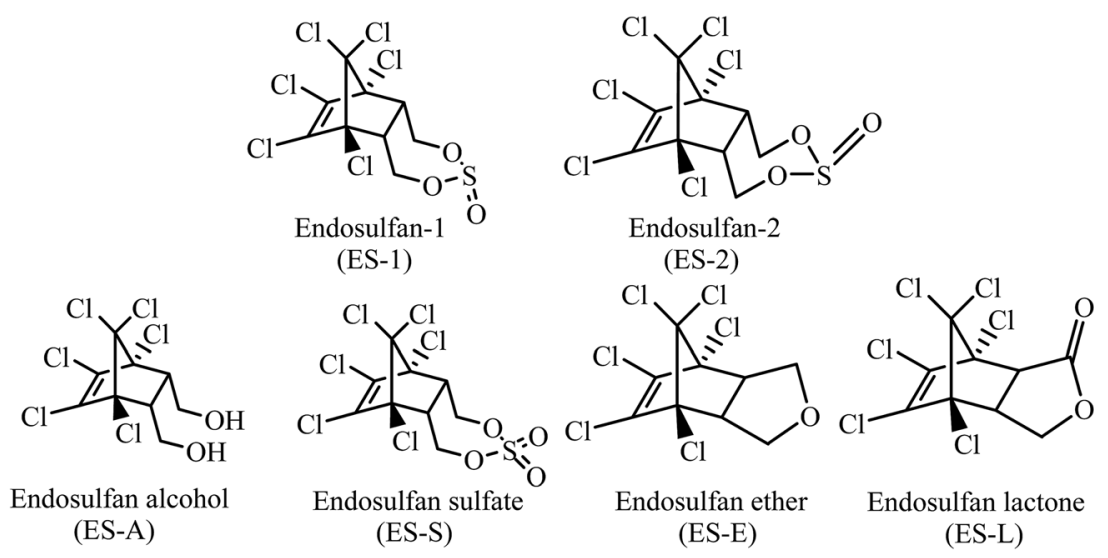

Fig. 1 Chemical structures of ES-isomers and metabolites.

Based on the above information it may be hypothesized that dechlorination of ES-isomers and ES-metabolites produces degradation products, which owing to their lower chlorine content may be less toxic and more amenable to subsequent biodegradation and eventual mineralization. Hence, there is a need to study the methods for dechlorination of ES-isomers and ES-metabolites, identify the resultant degradation products and screen the identified degradation products for toxicity and biodegradation potential.

Zero-valent iron (ZVI) has been used as an effective agent for dechlorination of many toxic organic compounds. ${ }^{24-29}$ Nanoscale zero valent iron (NZVI) particles are generally more effective than micron-scale zero valent iron (MZVI) particles for degradation of pollutants. ${ }^{30-32} \mathrm{ZVI}$ interaction with chlorinated organic compounds in reducing environment leads to dechlorination either via beta elimination or sequential elimination of chlorine molecules from the chlorinated compound..$^{33}$ Many other metals, namely $\mathrm{Pd}^{0}, \mathrm{Ni}^{0}, \mathrm{Mg}^{0}$ etc. and bi-metallic alloys, such as $\mathrm{Ni}^{0}-\mathrm{Fe}^{0}$ or $\mathrm{Pd}^{0}-\mathrm{Fe}^{0}$ etc. particles have also been used for reductive dechlorination of chlorinated pollutants. ${ }^{34,35}$ Bimetallic nano-particles, i.e., NZVI doped with other trace metals have been used by many researchers for the degradation of chlorinated pollutants. ${ }^{36,37}$ The trace constituents, such as metals like palladium (Pd), platinum (Pt), silver $(\mathrm{Ag})$ or nickel (Ni) may enhance the reactivity of NZVI particles. The presence of a noble metal controls the passivation of iron surface besides acting as a catalyst, ${ }^{26,34,36-41}$ thus increasing the effectiveness of reductive dechlorination of HOCs.

The main objective of present study was to suggest a mechanism for reductive dechlorination of ES-isomers and ESmetabolites through interaction with $\mathrm{NZVI} / \mathrm{Mg}^{0}$. The main tasks involved monitoring of the evolution of degradation products during the interactions of ES-isomers and ESmetabolites with NZVI $\left(\mathrm{Fe}^{0}\right)$ and $\mathrm{Mg}^{0}$; tentative identification of some of the degradation products using mass spectroscopy, proton NMR and X-ray crystallography and chemical synthesis and characterization of some of the degradation products were also carried out.

\section{Materials \& methods}

\subsection{Chemicals and glassware}

Technical standards of ES-1, ES-2, ES-S, ES-A, ES-L, ES-E and 2,4,5,6-tetrachloro- $m$-xylene were obtained from Sigma-Aldrich, India. Technical grade ES were obtained from United Phosphorous Ltd., Ankleshwar, India. Thin layer chromatography in $7: 3$ hexane-acetone medium was performed to isolate ES-1 and ES-2 from technical grade ES. The purity of ES-1 and ES-2 thus isolated was $\sim 99 \%$ when checked against the standards using GC-MS.

Solvents used for sample preparation and extraction, i.e., ethyl acetate, $n$-hexane, acetonitrile and acetone ( $>99 \%$ purity, HPLC grade) were procured from Merck, India. Sodium hydroxide pellets (98\%), anhydrous sodium sulfate (99\% purity), ferric chloride heptahydrate (96\% purity), sodium borohydride ( $97 \%$ purity), soluble starch (>99.5\% purity), $\mathrm{Mg}^{0}$ metal powder (99\%), glacial acetic acid (99.7\% purity), and hydrochloric acid (AR Grade) were procured from Loba Chemicals, India; sodium sulfate anhydrous (99\%) and zinc sulfate (99\%) were obtained from Thomas Baker Chemical Limited, India. Triethyl amine (>98\% purity), methane sulfonyl chloride (mesyl chloride) (>99\% purity) and $\mathrm{ZnCl}_{2}$ (95\% purity) were purchased from Fisher Scientific, India; Na metal cubes were procured form Sigma-Aldrich, India; NZVI was synthesized and characterized as described in earlier studies. ${ }^{\mathbf{4 2 , 4 3}}$

Borosilicate glass vials $(40 \mathrm{~mL})$ equipped with screw caps and Teflon faced re-sealable septa were used for various experiments. GC vials of $2 \mathrm{~mL}$ capacity with $11 \mathrm{~mm}$ PTFE rubber lined aluminium seal were used for the storage of extracted samples prior to analysis. Both types of vials were procured from Wheaton Science, Millville, NJ, USA.

\subsection{Experiments with NZVI}

The chemical structures of ES-isomers and ES-metabolites used in this study are as shown in Fig. 1. Concentrated stock solutions of ES-1, ES-2, ES-S, ES-L, ES-E and ES-A were prepared in acetone. For experiments with each compound, $40 \mathrm{~mL}$ vials was filled with $0.1 \mathrm{~g} \mathrm{~L}^{-1}$ aqueous NZVI suspension and the required 
volume of stock solution was added to each vial. All such vials were capped tightly and put on a rotating shaker at $30 \mathrm{rpm}$ for mixing; control vials with no NZVI were also prepared. All operations were carried out in a glove box and in an atmosphere of nitrogen. Vials were removed in duplicate (along with one control) at specified time intervals for analysis. Ambient temperature was approximately $31 \pm 3{ }^{\circ} \mathrm{C}$ and the $\mathrm{pH}$ maintained between $8.5-8.9$ in these vials.

\subsection{Synthesis of endosulfan ether}

In addition to ES-1 and ES-2, ES-E was also required in substantial quantities during this study. ES-E was synthesized from technical grade ES by a two-step process as shown in Fig. 2 . In the first step, $5 \mathrm{~g}$ of technical grade ES was dissolved in $50 \mathrm{~mL}$ of $1: 1$ acetone-water mixture. Next, $0.1 \mathrm{~mol}$ of $\mathrm{NaOH}$ dissolved in $10 \mathrm{~mL}$ of deionised water was added to the mixture followed by stirring for 12 hours at $40{ }^{\circ} \mathrm{C}$. The resulting mixture was extracted twice with $100 \mathrm{~mL}$ aliquots of ethyl acetate. Ethyl acetate was evaporated at $60{ }^{\circ} \mathrm{C}$ under high vacuum, leaving a white residue. This residue was purified by column chromatography and eluted with ethyl acetate/hexane. The solvent was evaporated yielding $3.55 \mathrm{~g}$ of white powder, which was confirmed to be $97 \%$ pure ES-A by GC-ECD analysis. In the next step, $2.0 \mathrm{~g}$ ES-A powder was dissolved in $50 \mathrm{~mL}$ of chloroform containing $7.0 \mathrm{mmol}$ of triethylamine and the solution was stirred for 1 hour at room temperature $\left(27 \pm 3{ }^{\circ} \mathrm{C}\right)$. Thereafter $7.0 \mathrm{mmol}$ of mesyl chloride was added slowly to the solution, followed by further stirring for 16 hours at room temperature. The organic phase was washed using $\mathrm{NaHCO}_{3}$ solution and dried under high vacuum. The dried white crystalline solid obtained was further purified by column chromatography and extracted with mixture of ethyl acetate and hexane. The solvent was evaporated yielding $1.63 \mathrm{~g}$ of white powder, which was confirmed to be $98 \%$ pure ES-E by GC-ECD analysis in comparison with ES-E standard.

\subsection{Experiments with $\mathrm{Mg}^{\mathrm{0}}$ and $\mathrm{Na}^{\mathrm{o}}$}

Experiments involving interaction between ES-E and $\mathrm{Mg}^{0}$ were carried out in $40 \mathrm{~mL}$ vials as per established procedure ${ }^{35} 2 \mathrm{~g}$ $\mathrm{Mg}^{0}$ powder, $50 \mathrm{mg} \mathrm{L}^{-1} \mathrm{ZnCl}_{2}, 4 \mathrm{~mL}$ of acetone and $1 \mathrm{mg} \mathrm{L}^{-1}$ of ES-E were added to the vial and the solution volume made up to $20 \mathrm{~mL}$ with deionised water. $0.5 \mathrm{~mL}$ of glacial acetic acid was slowly added to the mixture with constant manual shaking. The vials were tightly capped and after 30 minutes, were put on the rotor at $30 \mathrm{rpm}$ for 2 hours. Vials were removed in duplicate (along with one control) at various time intervals for analysis.
Ambient temperature was approximately $31 \pm 3{ }^{\circ} \mathrm{C}$. For experiments involving interaction between ES-E and $\mathrm{Na}^{0}, 1.5 \mathrm{~g}$ ES-E was dissolved in $50 \mathrm{~mL}$ of ethanol and refluxed with $0.4 \mathrm{~mol}$ of $\mathrm{Na}^{0}$ metal for three hours at $100{ }^{\circ} \mathrm{C}$.

\subsection{Extraction methods}

The procedure for the extraction of ES-isomers and ESmetabolites for GC and GC-MS analysis was adapted from Singh and Bose (2016). ${ }^{43}$ In brief, the aqueous samples were mixed with ethyl acetate (1:4 ratio) and $250 \mathrm{mg} \mathrm{NaCl}$ in tightly capped vials and mixed on a vortex mixer (Vertex Genie-2, Scientific Industries, USA) for 10 minutes. The contents were then centrifuged (BiofugeStratos, Heraeus, Germany) at $3000 \times$ $g$ for phase separation. After extraction, the samples were passed through anhydrous sodium sulfate to remove moisture. These were stored in $2 \mathrm{~mL}$ autosampler vials for analysis in GCECD and GC-MS. The percent recoveries of ES-1, ES-2, ES-S, ESA, ES-A and ES-E by using the above extraction procedure were $98.6 \pm 11.3 \%, 97.4 \pm 6.6 \%, 94.2 \pm 5.9 \%, 92.01 \pm 7.3 \%, 93.1 \pm$ $7.1 \%$ and $94.2 \pm 4.8 \%$, respectively. These recoveries were comparable to those obtained by the soxhlet extraction procedure (USEPA Method 3540).

\subsection{Analytical methods}

A Gas Chromatograph (GC) was used for analysis (Model Clarus 500, Perkin-Elmer, USA), equipped with an electron capture detector (ECD) and a capillary column (MXT-5) of length $30 \mathrm{~m}$, internal diameter $0.25 \mathrm{~mm}$ and film thickness $0.25 \mu \mathrm{m}$. The internal standard used was 2,4,5,6-tetrachloro- $m$-xylene. Nitrogen was used as the carrier gas at $68.95 \mathrm{kPa}(10 \mathrm{psi})$ and as

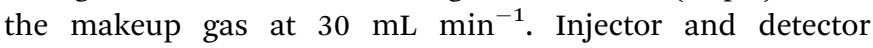
temperatures were $250{ }^{\circ} \mathrm{C}$ and $375{ }^{\circ} \mathrm{C}$, respectively. The oven temperature program was as follows: start at $120{ }^{\circ} \mathrm{C}$, hold for 1 minute; increase to $170{ }^{\circ} \mathrm{C}$ (a) $10{ }^{\circ} \mathrm{C} \mathrm{min}^{-1}$, hold for 8 minutes; increase to $220^{\circ} \mathrm{C}$ @ $18{ }^{\circ} \mathrm{C} \mathrm{min}{ }^{-1}$, hold for 8 minutes; increase to $240{ }^{\circ} \mathrm{C}$ @ $12{ }^{\circ} \mathrm{C} \min ^{-1}$ and hold for 5 minutes.

GC-MS analysis was done by Ion trap GC-MS (Polaris Q, ThermoFinnigan, USA) using $30 \mathrm{~m} \times 0.25 \mathrm{~mm} \times 0.25 \mathrm{~mm}$ DB5MS and DB-XLB capillary columns (Agilent Technologies, India). The career gas was helium at a flow rate of $1 \mathrm{~mL} \mathrm{~min}{ }^{-1}$. Injector, ion source and auxiliary line temperatures were 250, 200 and $260{ }^{\circ} \mathrm{C}$, respectively. Oven temperature program was as follows: start at $120{ }^{\circ} \mathrm{C}$, increase to $180{ }^{\circ} \mathrm{C}$ @ $8{ }^{\circ} \mathrm{C} \mathrm{min}^{-1}$, hold for 10 minutes; increase to $220^{\circ} \mathrm{C}$ @ $10^{\circ} \mathrm{C} \mathrm{min}{ }^{-1}$ and hold for 10 minutes. Ionization was carried out at $70 \mathrm{eV}$. The analysis for each sample was done in the full scan mode $(50-500 \mathrm{~m} / \mathrm{z})$ as well

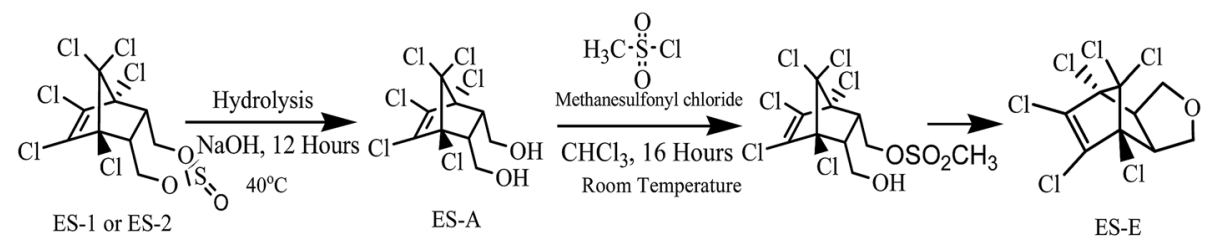

Fig. 2 Synthesis of (a) ES-E and $(b-d)$ dechlorinated degradation products of ES-E. 
as in the MS/MS mode. The GC-MS/MS details were adopted from Tiwari and Guha (2013). ${ }^{44}$ A different oven temperature program was used for lower dechlorinated by-products analysis. The temperature program was as follows: start at $70{ }^{\circ} \mathrm{C}$, hold for 10 minutes; increase to $180{ }^{\circ} \mathrm{C}$ (a) $8{ }^{\circ} \mathrm{C} \mathrm{min}^{-1}$, hold for 5 minutes; increase to $220{ }^{\circ} \mathrm{C}$ (a) $10{ }^{\circ} \mathrm{C} \min ^{-1}$ and hold for 12 minutes.

\section{Results \& discussion}

\subsection{Reductive dechlorination by NZVI}

The evolution of degradation products during reductive dechlorination of ES-isomers, i.e., ES-1 and ES-2, and ESmetabolites, namely ES-S, ES-L, ES-E and ES-A, by NZVI was recorded by a time series of chromatograms corresponding to each experiment. The data presentation scheme is summarized in Table 1. In Table 1, the parent compounds are referred by abbreviations used earlier, i.e., ES-1, ES-2, etc. The degradation products are denoted as ES- $1_{x}$, where the suffix ' $x$ ' corresponds to order of appearance of the degradation product peaks during the dechlorination process shown in the gas chromatogram (Fig. 3b). For example, ES- $\mathbf{1}_{2}$ denotes the degradation product which appeared second during the interaction of ES-1 with NZVI. Only the following information is presented for each experiment; (a) chromatogram at the start of the experiment showing the peak corresponding to the parent molecule; (b) chromatogram at an intermediate time during the experiment, where the peaks corresponding to the parent molecule and all degradation products are simultaneously visible; (c) mass spectra of the parent compound and corresponding reference spectra from the NIST library; (d) mass spectra corresponding to each degradation product peak and the corresponding proposed chemical structure. The location information, i.e., figure/table numbers for the above data for each experiment are given in Table 1.

\subsection{Cyclization of endosulfan alcohol}

The analysis of ES-A and its dechlorinated by-products was difficult in GC-MS due to the presence of hydroxyl group. However, ES-A and its dechlorination products could be converted to their corresponding ethers by the procedure shown in Fig. 2. Thus, the peak elution times and mass spectra of ES-A and its dechlorination products as shown in Fig. SI5† were identical to those obtained in case of ES-E (Fig. SI $4 \dagger$ ).

The variation in $\mathrm{pH}$ during NZVI experiments was between 7.5-8.8, and at a higher pH, hydrolysis of ES-1 and ES-2 to ES-A is reported..$^{\mathbf{1 3 , 1 4 , 4 5}}$ In the present experiments, ES-1, ES-2 and its dechlorination products disappeared from the chromatograph after 48 hours. However, ES-A and its dechlorination products could still be detected in these solutions after conversion of ESA and its dechlorination products to their corresponding ethers. Moreover, these dechlorination products were identical to those detected during dechlorination of ES-E. So the dechlorination products obtained from the dechlorination experiments of ES-1, ES-2, ES-A and ES-E were identical, which supports the hypothesis of a similar dechlorination sequence for ES skeleton, irrespective of the particular compound being dechlorinated. Considering the central role of ES-E as described above and the fact that ES-E does not hydrolyze, it was selected as the representative compound for further experiments.

\subsection{Reductive dechlorination of ES-E by $\mathrm{Mg}^{\mathrm{0}}$ and $\mathrm{Na}^{\mathrm{o}}$}

The degradation products formed during reductive dechlorination of ES-E by $\mathrm{Mg}^{0}$ were all observed in a chromatogram

Table 1 Interaction of ES and ES-metabolites with NZVI: data summary ${ }^{a}$

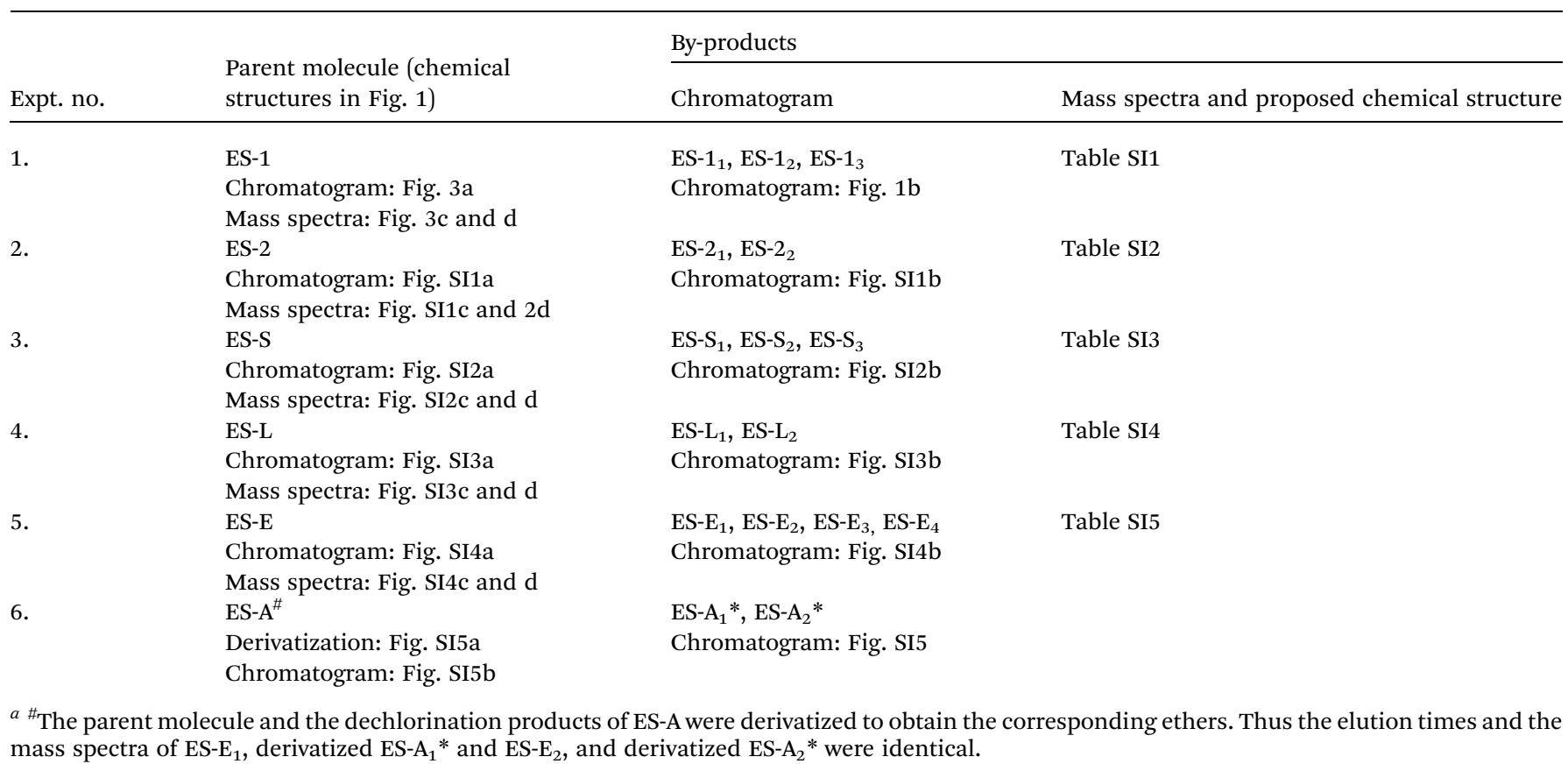

$a{ }^{\#}$ The parent molecule and the dechlorination products of ES-A were derivatized to obtain the
mass spectra of ES- $\mathrm{E}_{1}$, derivatized ES- $\mathrm{A}_{1}{ }^{*}$ and ES- $\mathrm{E}_{2}$, and derivatized ES- $\mathrm{A}_{2}{ }^{*}$ were identical. 

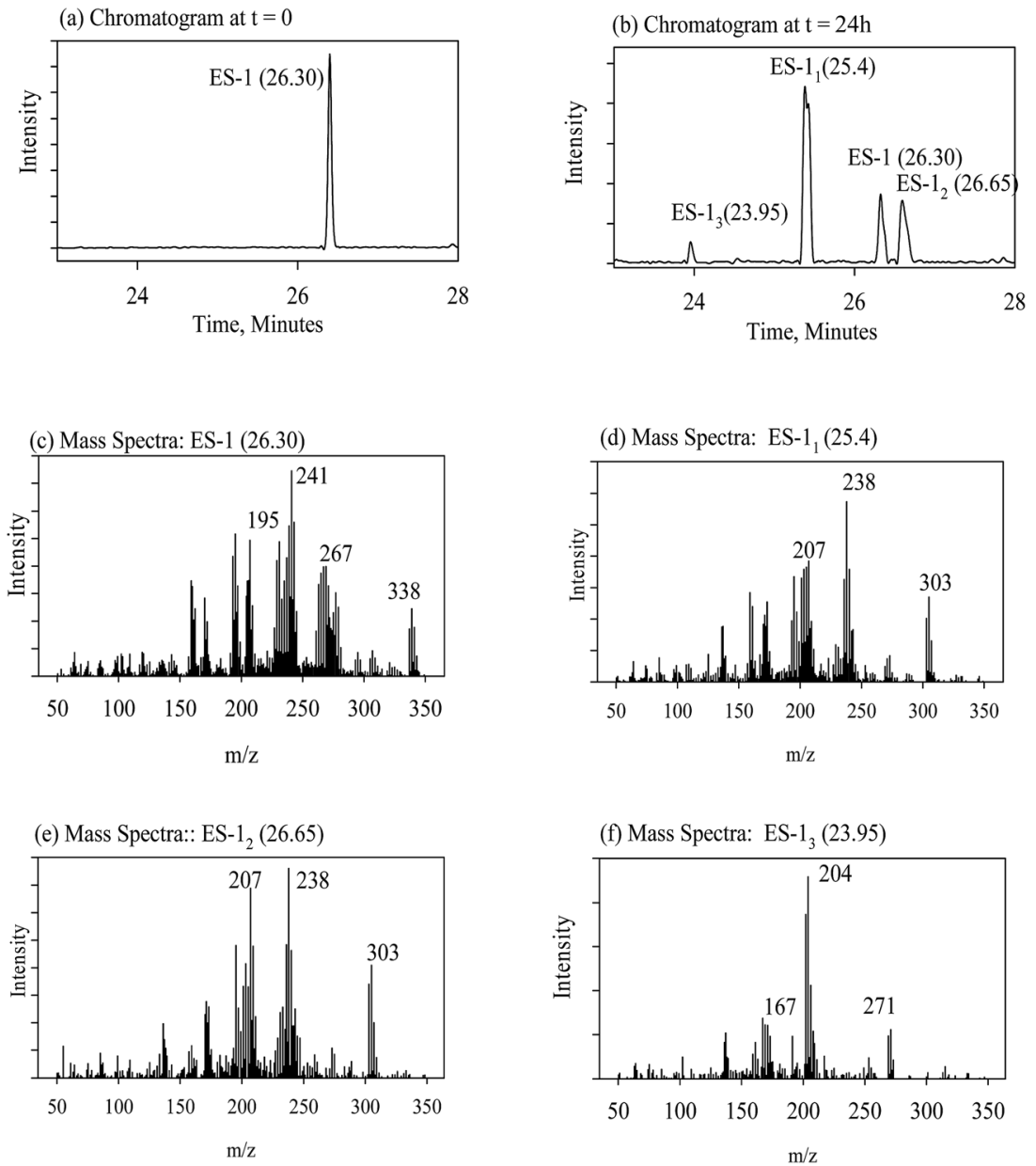

Fig. 3 Reductive dechlorination of ES-1 by NZVl; (a) chromatogram at, $t=0$; (b) chromatogram at, $t=24$ h; (c) mass spectra of ES-1; (d) mass spectra of ES-1 $1_{1} ;(e)$ mass spectra of ES-1 $1_{2} ;(f)$ mass spectra of ES-1 $1_{3}$.

obtained from a sample extracted at an intermediate point during the experiment (Fig. 4b). Based on this chromatogram and the associated mass spectra, the following general observations were possible regarding formation of degradation products during the reductive dechlorination of ES-E by $\mathrm{Mg}^{0}$, (a) reductive dehalogenation resulted in the formation of six degradation products, ES- $\mathrm{E}_{1 \mathrm{a}}$, ES- $\mathrm{E}_{3 \mathrm{a}}-\mathrm{ES}-\mathrm{E}_{7 \mathrm{a}}$ (Fig. 4b); (b) mass spectra of ES-E $\mathrm{E}_{1 \mathrm{a}}, \mathrm{ES} \mathrm{E}_{3 \mathrm{a}}$ and ES-E $\mathrm{E}_{4 \mathrm{a}}$ (Table SI6†) were consistent with the loss of one, two and three chlorine atoms, respectively from the parent compound and were nearly identical to the mass spectra of ES-E $E_{1}$, ES-E $_{3}$ and ES- $E_{4}$ (Table SI5 $\dagger$ ) obtained earlier during reductive dechlorination of ES-E by NZVI; (c) mass spectra of ES- $E_{5 a}$, ES- $_{6 a}$ and ES-E $\mathrm{E}_{7 \mathrm{a}}$ were consistent with the loss of four, five and six chlorine atoms, respectively from the parent compound (Table SI6†).

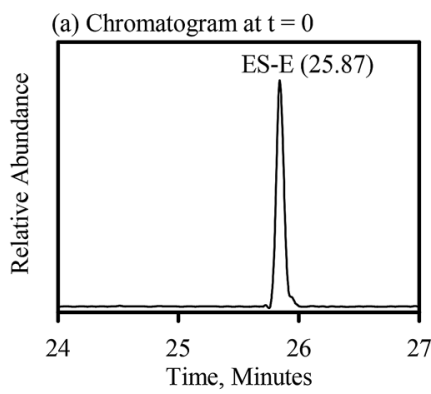

(b) Chromatogram at $\mathrm{t}=2 \mathrm{~h}$ by $\mathrm{Mg}^{0}$

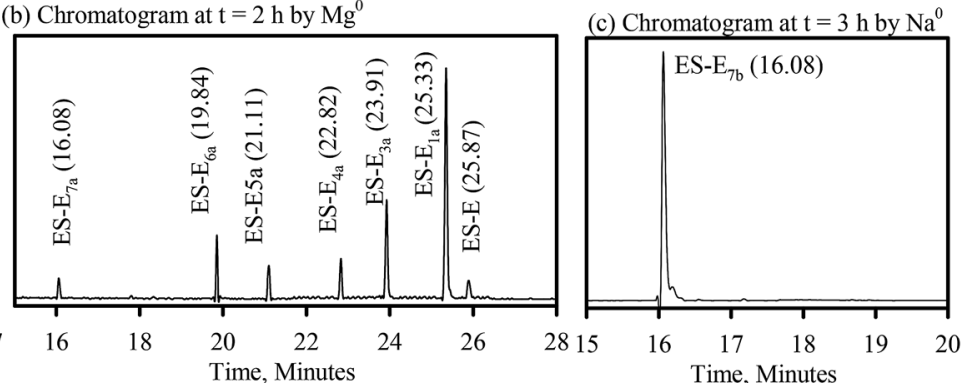

Fig. 4 Reductive dechlorination of ES-E by $\mathrm{Mg}^{0}$; (a) chromatogram at, $t=0$; (b) chromatogram at, $t=2 \mathrm{~h}$ by $\mathrm{Mg}^{0}$; (c) chromatograph at $t=3 \mathrm{~h}$ by $\mathrm{Na}^{0}$. 
Only one degradation product (ES-E $\left.\mathrm{E}_{7 \mathrm{~b}}\right)$, consistent with the loss of six chlorine atoms from the parent compound, was observed during reductive dechlorination of ES-E by $\mathrm{Na}^{0}$ (Fig. 4c) and confirmed by NIST library (NIST no. 105262) as endo-4-oxatricyclo[5.2.1.0(2.6)]dec-8-ene. The mass spectra of ES-E $_{7 \mathrm{~b}}$ (Table SI7 $\dagger$ ) was nearly identical to the mass spectra of ES-E $_{7 a}$ (Table SI6 $\dagger$ ) obtained earlier during reductive dechlorination of ES-E by $\mathrm{Mg}^{\mathrm{O}}$.

\subsection{Dechlorination mechanism}

In all experiments involving reductive dechlorination of ES and its metabolites by NZVI, two distinct degradation products were initially observed. Both had mass spectra analogous to the loss of one chlorine atom from the parent compound (Tables SI1$5 \dagger$ ). In some cases, other degradation products, having mass spectra consistent with the loss of two or three chlorine atoms from the parent compound, were also observed sequentially. The experiment involving reductive dechlorination of ES-E by $\mathrm{Mg}^{0}$ showed sequential formation of degradation products having mass spectra consistent with the loss of 1-6 chlorine atoms from the parent compound, i.e., $\mathrm{ES}-\mathrm{E}_{1 \mathrm{a}}, \mathrm{ES}_{-} \mathrm{E}_{3 \mathrm{a}}-\mathrm{ES}_{-} \mathrm{E}_{7 \mathrm{a}}$. In this case, only one degradation product (ES- $\mathrm{E}_{1 \mathrm{a}}$ ) was observed with mass spectrum corresponding to the loss of a single chlorine atom. It is possible that a degradation product similar to ES-E $E_{2}$ (with NZVI) was also formed in this case, but was quickly further dechlorinated due to vigorous interaction with $\mathrm{Mg}^{\mathrm{O}}$ and thus escaped observation.

It is increasingly apparent that the mechanisms for reductive dechlorination in the above experiments with NZVI and $\mathrm{Mg}^{0}$ are essentially the same, i.e., dechlorination occurs sequentially, but may or may not proceed to the dechlorinated end product depending on the intensity of the reducing conditions employed. Reductive dechlorination appeared to be through a surface mediated single electron transfer mechanism. Such a mechanism is consistent with the observed experimental data and has also been proposed by several researchers for the reductive dechlorination of other organic pollutants. ${ }^{32,46-48}$

The reductive dechlorination of ES-E by $\mathrm{Na}^{0}$ showed only one degradation product having mass spectra consistent with the loss of all six chlorine atoms from the parent molecule. The dechlorination process in this case was very rapid and it is possible that the dechlorination mechanism probably involved simultaneous attack on all chlorinated sites by electron shuttles transporting electrons from the Na surface to the ES-E molecule. Such a mechanism is consistent with the observed rapid and near simultaneous dechlorination at all sites.

\subsection{Chemical structure of degradation products}

Considering the ES-skeleton shown in Fig. 5, it is apparent that $\mathrm{Fe}^{0} / \mathrm{Mg}^{0}$-mediated sequential reductive dechlorination can start with the removal of chlorine atoms at positions 1,2 or 3 . The formation of two mono-dechlorinated products with the same mass spectra (Fig. 3d and e), suggests that the initial attack takes place on position 1 and it can be assumed that dechlorination can start at positions $\mathbf{1 a}$ or $\mathbf{1 b}$. Since the chemical environment of the newly attached hydrogen atom will be

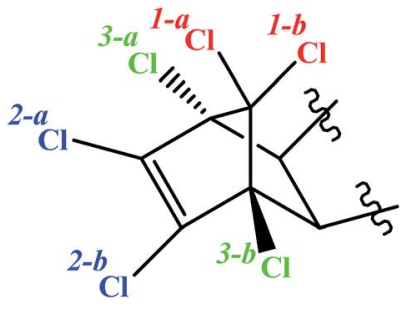

Fig. 5 Structure of ES-skeleton.

different depending on whether it is attached at position 1a or $\mathbf{1 b}$, the resulting dechlorinated isomers are likely to have different physical properties and hence these are expected to appear as separate peaks in chromatograms. This was indeed observed in this study in experiments with NZVI; third, dechlorination commencing at positions $2 \mathbf{a}$ or $\mathbf{2 b}$ and $\mathbf{3 a}$ or $\mathbf{3 b}$ will result in the formation of enantiomers, which will not be seen as separate peaks in chromatograms; fourth, we may assume that the initial dechlorination occurs randomly at positions either 1,2 or 3 . However, in that case, the second dechlorination step will result in the formation of several degradation products consistent with the loss of two chlorine atoms from the parent molecule. However, in all the above experiments, only a single degradation product was observed in accordance with the loss of two chlorine atoms from the parent molecule.

On the basis of the above reasoning, it is apparent that the second dechlorination step results in the loss of chlorine atoms at both positions 1a and $\mathbf{1 b}$. Further, the third dechlorination step is likely to occur at position $\mathbf{2 a}$ or $\mathbf{2 b}$ for the following reasons; the carbon atom at site $\mathbf{3 a}$ or $\mathbf{3 b}$ is at a saturated bridge position of a bicyclic compound, rendering electron transfer to the attached chlorine atom very difficult; a study on electrochemical dechlorination of ES indicated that dechlorination at position $\mathbf{3 a}$ and $\mathbf{3 b}$ is not favored; ${ }^{49}$ analogy with a study on reductive dechlorination of 1,2-dichloroethene ${ }^{50}$ also indicates that dechlorination at position 2 is favored. As mentioned earlier, the two products formed due to the third dechlorination step are enantiomers and will hence appear as a single peak in the chromatogram. Fourth dechlorination step would results in the removal of chlorine atoms from both $2 \mathbf{a}$ and $2 \mathbf{b}$ positions. The fifth dechlorination step is expected to occurs at position 3a or $\mathbf{3 b}$ again resulting in the formation of two enantiomers and the final dechlorination step thereafter results in the formation of the fully dechlorinated product. The tentative chemical structures for the various degradation products observed during this study have been proposed in consonance with the dechlorination sequence as discussed above and the corresponding mass spectra (for degradation products of ES-1, ES-2, ES-S, ES-L, see Tables SI1-3 and $5, \dagger$ respectively; for degradation products of ES-E, see Tables SI4, 6 and $7 \dagger$ ). Although the exact pathway for all compounds (ES-isomer and metabolites) is matter of investigation with more experimental evidence. 


\subsection{Synthesis of dechlorinated by-products}

Dechlorination of the ES-skeleton (Fig. 5) can commence at positions 1, 2 or 3. The preponderance of evidence discussed in the previous section suggests that dechlorination commences at position 1. Unequivocal determination of the first dechlorination position is critical, since the proposed chemical structures of the degradation products hinges on this determination. An unequivocal confirmation of the initial dehalogenation position requires the synthesis and subsequent analysis of the actual dechlorinated products. As noted earlier, there is a strong possibility that the degradation pathway of ES-isomers and ESmetabolites are similar. Further, considering the central role of ES-E as discussed earlier, it was decided that the degradation mechanism of ES-E will be studied in detail. Thus, some of the degradation products of ES-E formed through reductive dechlorination were synthesized.

The first dechlorinated product synthesized corresponded to the loss of one chlorine atom from the parent molecule. The procedure for this synthesis is summarized in Fig. 6a. First, $2 \mathrm{~g}$ of ES-E was dissolved in $30 \mathrm{~mL}$ of acetone-water mixture $(1: 1)$ followed by the addition of $5.0 \mathrm{~g}$ of $\mathrm{Mg}^{0}$ metal powder and $5.0 \mathrm{mmol}$ of $\mathrm{ZnCl}_{2}$. Then, $5 \mathrm{~mL}$ of glacial acetic acid was added dropwise with constant stirring. The mixture was stirred for another 2 hours for completion of the reaction at room temperature $\left(27 \pm 3{ }^{\circ} \mathrm{C}\right)$. The degradation products thus formed were extracted twice with $50 \mathrm{~mL}$ of ethyl acetate. The solvent in the extract was dried at $60{ }^{\circ} \mathrm{C}$ under high vacuum. The required product was purified by column chromatography and eluted with ethyl acetate/hexane. Evaporation of the solvent yielded $1.51 \mathrm{~g}$ of a white crystalline powder. The fact that this compound was consistent with the loss of one chlorine atom from the parent compound was confirmed by GC-MS and ${ }^{1} \mathrm{H}$ NMR (500 MHz, DMSO-d $\left.6,25{ }^{\circ} \mathrm{C}, \mathrm{TMS}\right)$.
The second dechlorinated product synthesized corresponded to the loss of two chlorine atoms from the parent ES-E molecule. The starting material for this synthesis was $300 \mathrm{mg}$ of the first dechlorinated product. The procedure for this synthesis is summarized in Fig. 6b. However, the quantities of the reagents used were modified proportionately to account for the lower amount of starting material used in this case. Purification of the product was done on TLC plates using mixture of acetone hexane as eluting solvent. The fact that this compound was consistent with the loss of two chlorine atoms from the parent molecule was confirmed by GC-MS analysis.

The third dechlorinated product synthesized corresponded to the loss of all six chlorine atoms from the parent molecule. The procedure of synthesis is summarized in Fig. 6c. $1.5 \mathrm{~g}$ ES-E was dissolved in $50 \mathrm{~mL}$ of ethanol and refluxed with $0.4 \mathrm{~mol}$ of $\mathrm{Na}^{0}$ metal for three hours at $100{ }^{\circ} \mathrm{C}$. The cooled mixture was mixed with crushed ice and then extracted with $100 \mathrm{~mL}$ ethyl acetate. The solvent was evaporated at $45{ }^{\circ} \mathrm{C}$ under high vacuum..$^{51}$ The resulting product was purified on TLC plates using mixture of acetone and hexane as eluting solvent. The fact that this compound was consistent with removal of all chlorine atoms from the parent molecule was confirmed by GC-MS and the analogy of its mass spectra with the corresponding mass spectra available in NIST library.

\subsection{Experimental evidence for a plausible degradation pathway}

The theoretical ${ }^{1} \mathrm{H}$ NMR for singly dechlorinated ES-E was obtained assuming that dechlorination occurred from position 1 , 2 or 3 as shown in Fig. SI6. $\dagger$ The actual ${ }^{1} \mathrm{H}$ NMR of ES-E and the dechlorinated product ES- $\mathrm{E}_{1 \mathrm{a}}$ are shown in Fig. SI7. $\dagger$ The ${ }^{1} \mathrm{H}$ NMR of the degradation product ES- $\mathrm{E}_{1 \mathrm{a}}$ provides the position for introduction of new proton at $\delta$ (PPM) 4.28, which is very (a)

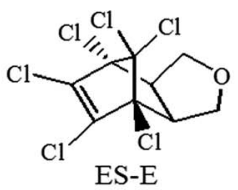

(b)

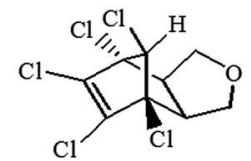

First dechlorinated product

(c)

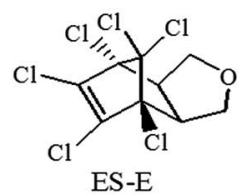

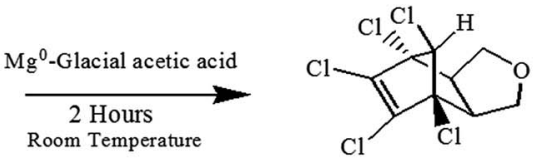

1,7,8,9,10-Pentachloro-4-oxa-tricy clo[5.2.1.0 $\left.0^{2,6}\right] \mathrm{dec}-8$-ene

First dechlorinated product

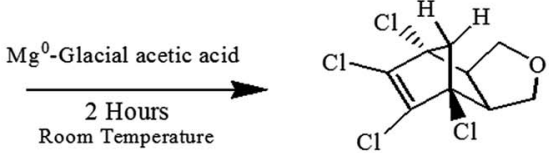

1,7,8,9-Tetrachloro-4-oxa-tricyclo[5.2.1.0 $\left.0^{2,6}\right]$ dec-8-ene

Second dechlorinated product

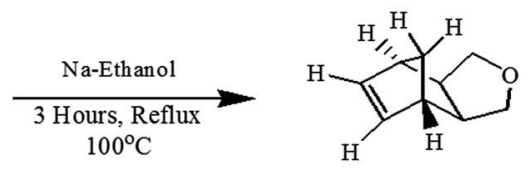

4-Oxa-tricyclo[5.2.1.0 $\left.0^{2,6}\right]$ dec-8-ene

Fully dechlorinated product

Fig. 6 Synthesis of (a) first dechlorinated product, (b) second dechlorinated product and (c) fully dechlorinated product of ES-E. 
close to the $\delta$ (PPM) 4.48 value obtained from the theoretical ${ }^{1} \mathrm{H}$ NMR corresponding to the proton insertion at position 1 .

Further confirmation of position of dechlorination either from 1a or 1b was obtained via single crystal X-ray diffraction measurements mounted on a CCD Bruker SMART APEX diffractometer. The crystallographic figure has been generated using Diamond $3.2 \mathrm{~g}$ (Fig. SI8 $\dagger$ ). The contribution of all hydrogen atoms has been incorporated in both empirical formulas and formula weights of the complexes. X-ray diffraction measurements confirmed that the first dechlorination takes place from $\mathbf{1 b}$ position (Fig. SI8†).

All evidence presented suggest that dechlorination mechanism for all ES and ES-metabolite molecules through interaction with $\mathrm{NZVI} / \mathrm{Mg}^{0}$ surface was identical. Based on the above results, the most probable pathway for the dechlorination process was proposed. Dechlorination seems to occurs sequentially, yielding products $1 \mathbf{a} / \mathbf{1} \mathbf{b}, 2,3 \mathbf{a} / \mathbf{3 b}, \mathbf{4}, 5 \mathbf{a} / \mathbf{5 b}$ and 6 (Fig. 7). Products $\mathbf{1 a}$ and $\mathbf{1 b}$ are isomers and hence observed as separate peaks in chromatograms. Products $\mathbf{3 a} / \mathbf{3 b}$ and $\mathbf{5 a} / \mathbf{5 b}$ are enantiomers and hence appeared as the overlapping peaks in chromatogram. Peaks corresponding to intermediate products 2, 4 and the dechlorinated product 6 were also observed. The proposed pathway (Fig. 7) is a tentative pathway and exact pathway is matter of future investigation, as $3^{\text {rd }}, 4^{\text {th }}$ and $5^{\text {th }}$ dechlorination needs more experimental supports.

The extent of dechlorination of the various ES-isomers and ES-metabolites appeared to depend on the intensity of the reducing conditions employed; hence, dechlorination was more complete during interaction with the $\mathrm{Mg}^{0}$ surface. The rate of dechlorination was however, different for different molecules depending on the nature of the side-chain attached to the ES skeleton shown in Fig. 5.

\section{Conclusions}

Reductive dechlorination of ES and ES-metabolites through interaction with NZVI and $\mathrm{Mg}^{0}$ was investigated. During dechlorination of ES-isomers and ES-metabolites by NZVI, two distinct degradation products, both having mass spectra consistent with the loss of one chlorine atom from the parent compound, were initially observed. Other degradation products were observed later and had mass spectra consistent with the loss of two or three chlorine atoms from the parent compound. During dechlorination of ES-E by $\mathrm{Mg}^{0}$, degradation products having mass spectra consistent with the loss of 1-6 chlorine atoms from the parent compound were observed sequentially. The dechlorination mechanism for all ES-isomers and ESmetabolite molecules through interaction with $\mathrm{NZVI} / \mathrm{Mg}^{\mathrm{0}}$ surface appeared to be the similar. It was proposed that the abstraction of chlorine atoms occurred sequentially from positions $\mathbf{1 a} / \mathbf{1} \mathbf{b}, \mathbf{2 a} / \mathbf{2} \mathbf{b}$ and $\mathbf{3 a} / \mathbf{3 b}$ of the ES skeleton shown in Fig. 5 . Chemical structures of various degradation products are proposed based on the above order of chlorine abstraction and mass spectra of the degradation products. Dechlorinated products were synthesized using ES-E as the parent molecule. ${ }^{1} \mathrm{H}$ NMR, X-ray diffraction measurements, and GC-MS analysis of the synthesized products provide the final proof of initial chlorine abstraction from position $\mathbf{1 b}$ as shown in Fig. 5 .

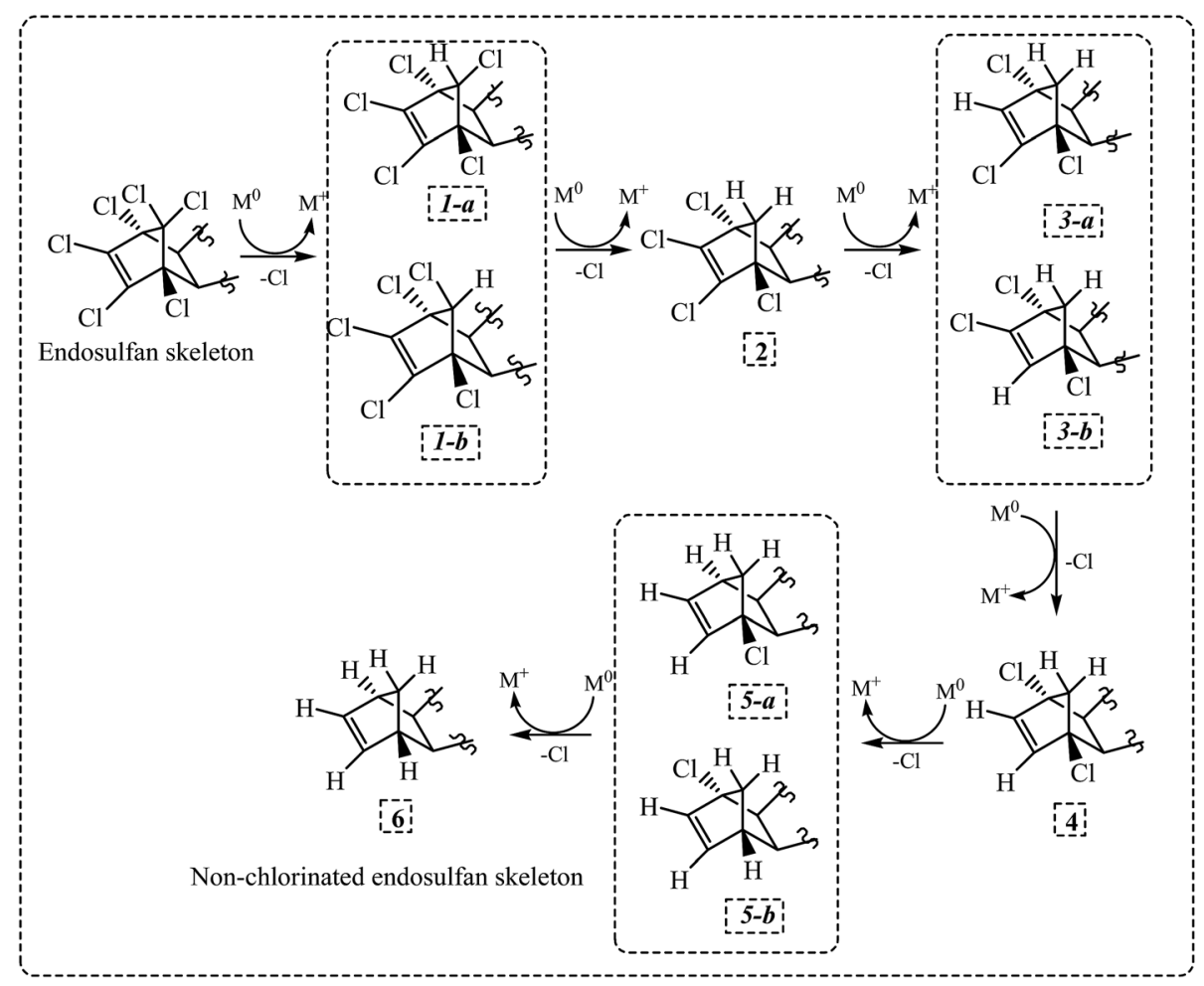

Fig. 7 Proposed dechlorination pathway for ES-isomers and metabolites. 
The extent of dechlorination of the various ES-isomers and ES-metabolites appeared to be variable and appeared to depend on the nature of the side-chain attached to the ES skeleton. Further, it appears that the extent of dechlorination could be enhanced by increasing the intensity of the reducing conditions employed. While using $\mathrm{Mg}^{0}$ as a reducing agent may be difficult in natural environment, application of bimetallic nanoparticles, i.e., $\mathrm{Ni}^{0}-\mathrm{Fe}^{0}$, or $\mathrm{Pd}^{0}-\mathrm{Fe}^{0}$ etc., may be an environmentally feasible option, which may result in more complete dechlorination of ES-isomers and ES-metabolites as compared to NZVI. Finally, the significance of the present study lies in the fact that partially or fully dechlorinated degradation products of ES and ES-metabolites may be less toxic and more amenable to subsequent biodegradation and ultimate mineralization in the natural environment. The toxicity study of these dechlorinated degradation products and their biodegradation potential requires further evaluation.

\section{Acknowledgements}

The authors thank Dr. Jitendra Kumar Bhatt and Dr. Narendra Mishra for their help in synthesis and solving crystal structure for new by-products.

\section{References}

1 Y. F. Li and R. W. Macdonald, Sci. Total Environ., 2005, 342, $87-106$.

2 J. Weber, C. J. Halsall, D. Muir, C. Teixeira, J. Small, K. Solomon, M. Hermanson, H. Hung and T. Bidleman, Sci. Total Environ., 2010, 408, 2966-2984.

3 R. I. M. Sunderam, D. M. H. Cheng and G. B. Thompson, Environ. Toxicol. Chem., 1992, 11, 1469-1476.

4 L. Beyger, R. Orrego, H. Guchardi and D. Holdway, Ecotoxicol. Environ. Saf., 2012, 76, 71-78.

$5 \mathrm{H}$. L. Henderson, J. Townsend and D. J. Tortonese, J. Endocrinol., 2008, 197, 343-350.

6 A. Caride, A. Lafuente and T. Cabaleiro, Toxicol. Lett., 2010, 197, 106-112.

7 O. Ozmen and F. Mor, Pestic. Biochem. Physiol., 2012, 102, 129-133.

8 C. J. Hapeman, L. L. McConnell, T. L. Potter, J. HarmanFetcho, W. F. Schmidt, C. P. Rice, B. A. Schaffer and R. Curry, Atmos. Environ., 2013, 66, 131-140.

9 R. Martens, Appl. Environ. Microbiol., 1976, 31, 853-858.

10 J. R. W. Miles and P. Moy, Bull. Environ. Contam. Toxicol., 1979, 23, 13-19.

11 T. F. Guerin, Environ. Pollut., 2001, 115, 219-230.

12 T. E. Archer, I. K. Nazer and D. G. Crosby, J. Agric. Food Chem., 1972, 20, 954-956.

13 S. M. Peterson and G. E. Batley, Environ. Pollut., 1993, 82, 143-152.

14 H. M. Shivaramaiah, F. Sanchez-Bayo, J. Al-Rifai and I. R. Kennedy, J. Environ. Sci. Health, Part B, 2005, 40, 711720.

15 C. N. G. Bedor, R. J. L. Morais, L. S. Cavalcanti, J. V. Ferreira and A. C. Pavão, Sci. Total Environ., 2010, 408, 6281-6284.
16 R. Martens, Bull. Environ. Contam. Toxicol., 1977, 17, 438446.

17 D. Henschler, Angew. Chem., Int. Ed. Engl., 1994, 33, 19201935.

18 G. Bonse, D. Henschler and P. J. Gehring, Crit. Rev. Toxicol., 1976, 4, 395-409.

19 A. Sato and T. Nakajima, Arch. Environ. Health, 1979, 34, 6975.

20 A. Bobra, W. Shiu and D. Mackay, in QSAR in Environmental Toxicology, ed. K. E. Kaiser, Springer Netherlands, 1984, ch. 2, pp. 3-16.

21 M. Zahedi-Tabrizi and R. Farahati, Comput. Theor. Chem., 2011, 977, 195-200.

22 K. Furukawa, K. Tonomura and A. Kamibayashi, Appl. Environ. Microbiol., 1978, 35, 223-227.

23 K. Furukawa, N. Tomizuka and A. Kamibayashi, Appl. Environ. Microbiol., 1979, 38, 301-310.

24 R. W. Gillham and S. F. O'Hannesin, Ground Water, 1994, 32, 958-967.

25 T. L. Johnson, M. M. Scherer and P. G. Tratnyek, Environ. Sci. Technol., 1996, 30, 2634-2640.

26 X.-q. Li and W.-x. Zhang, Langmuir, 2006, 22, 4638-4642.

27 R. A. Crane and T. B. Scott, J. Hazard. Mater., 2012, 211-212, 112-125.

28 S. P. Singh and P. Bose, RSC Adv., 2015, 5, 94418-94425.

29 B. I. Kharisov, H. V. Rasika Dias, O. V. Kharissova, V. Manuel Jimenez-Perez, B. Olvera Perez and B. Munoz Flores, RSC Adv., 2012, 2, 9325-9358.

$30 \mathrm{~J}$. Gotpagar, E. Grulke, T. Tsang and D. Bhattacharyya, Environ. Prog., 1997, 16, 137-143.

31 B. Deng, D. R. Burris and T. J. Campbell, Environ. Sci. Technol., 1999, 33, 2651-2656.

32 W. A. Arnold and A. L. Roberts, Environ. Sci. Technol., 2000, 34, 1794-1805.

$33 \mathrm{~S} . \mathrm{M}$. Cook, Assessing the use and application of zero-valent iron nanoparticle technology for remediation at contaminated sites, Jackson State University, 2009.

34 W.-J. Liu, T.-T. Qian and H. Jiang, Chem. Eng. J., 2014, 236, 448-463.

35 A. Begum and S. K. Gautam, Water Res., 2011, 45, 2383-2391. 36 T. Hua, L. Jinjun, M. Zhen, L. Landong and H. Zhengping, Sep. Purif. Technol., 2009, 66, 84-89.

37 T.-T. Lim, J. Feng and B.-W. Zhu, Water Res., 2007, 41, 875883.

38 F. He and D. Zhao, Environ. Sci. Technol., 2005, 39, 33143320.

39 B. Schrick, J. L. Blough, A. D. Jones and T. E. Mallouk, Chem. Mater., 2002, 14, 5140-5147.

40 S. Luo, S. Yang, X. Wang and C. Sun, Chemosphere, 2010, 79, 672-678.

41 Y. Cho and S.-I. Choi, Chemosphere, 2010, 81, 940-945.

42 S. P. Singh, P. Bose, S. Guha, S. K. Gurjar and S. Bhalekar, Chemosphere, 2013, 92, 811-820.

43 S. P. Singh and P. Bose, J. Chem. Technol. Biotechnol., 2016, 91, 2313-2321.

44 M. Tiwari and S. Guha, Environ. Monit. Assess., 2013, 185, 8451-8463. 
45 S. S. Walse, K. D. Shimizu and J. L. Ferry, Environ. Sci. Technol., 2002, 36, 4846-4853.

46 M. Elsner, S. B. Haderlein, T. Kellerhals, S. Luzi, L. Zwank, W. Angst and R. P. Schwarzenbach, Environ. Sci. Technol., 2004, 38, 2058-2066.

47 A. L. Roberts, L. A. Totten, W. A. Arnold, D. R. Burris and T. J. Campbell, Environ. Sci. Technol., 1996, 30, 2654-2659.
48 E. J. Bylaska, M. Dupuis and P. G. Tratnyek, J. Phys. Chem. A, 2008, 112, 3712-3721.

49 J. V. J. Gassmann and G. Adiwidjaja, Zeitschrift für Naturforschung B, 1996, 51, 417-420.

50 C. Nonnenberg, W. A. van der Donk and H. Zipse, J. Phys. Chem. A, 2002, 106, 8708-8715.

51 B. V. Lap and M. N. Paddon-Row, J. Org. Chem., 1979, 44, 4979-4981. 